\title{
Protocolo de rápida mejoría posoperatoria (RAMPO) en cirugía gastrointestinal
}

\author{
Protocol for fast postoperative improvement (FPOI) in gastrointestinal surgery
}

César Ramírez-Cáceres ${ }^{1,2 *}$, Estrella Uzcátegui-Paz y Ricardo Lozano-Hernández³

${ }^{1}$ Servicio de Cirugía General, Instituto Autónomo Hospital Universitario de Los Andes; ${ }^{2}$ Cátedra de Ciencias Morfológicas, Facultad de Medicina, Universidad de Los Andes; ${ }^{3}$ Cátedra de Fisiopatología, Facultad de Farmacia y Bioanálisis, Universidad de Los Andes. Mérida, Venezuela

\begin{abstract}
Resumen
Introducción: El tratamiento peroperatorio ha requerido protocolos multimodales que estimulen la evolución del paciente y acorten la estancia hospitalaria. Objetivo: Identificar el tipo de patología y la intervención realizada, y evaluar la efectividad del protocolo de rápida mejoría posoperatoria (RAMPO) en pacientes sometidos a cirugía electiva gastrointestinal. Método: Se evaluaron 122 pacientes; a un grupo de 61 se les aplicó el protocolo RAMPO y en el otro grupo de igual número de pacientes se siguieron protocolos tradicionales (controles). Resultados: Ambos grupos ingresaron por cáncer colorrectal, colostomía por patología benigna y cáncer gástrico. Las intervenciones realizadas fueron resecciones colorrectales (35.25\%), restitución del tránsito intestinal (29.51\%) y gastrectomía (15.57\%). En el grupo RAMPO, los valores de glucemia posoperatoria (116.49 vs. $167.08 \mathrm{mg} / \mathrm{dl}$ ) reflejaron un mejor control metabólico con menor estancia hospitalaria (5.49 vs. 14.11 días), con un mayor grado de satisfacción (91.80\% vs. 19.67\%) al compararse con el grupo control ( $p<0.005)$. Conclusión: El protocolo RAMPO presentó aspectos relevantes al manejo tradicional de los pacientes sometidos a cirugías electivas del tracto gastrointestinal, siendo seguro, aceptable en nuestro medio y con egreso hospitalario más rápido, mejorando de manera significativa la evolución de los enfermos, con menos complicaciones y un alto grado de satisfacción, sin aumentar la morbimortalidad.
\end{abstract}

PALABRAS CLAVE: Peroperatorio. Cirugía gastrointestinal. Tratamiento tradicional.

\begin{abstract}
Introduction: Peroperative treatment has required multimodal protocols that stimulate patient evolution and shorten hospital stay. Objective: Identify the type of pathology, intervention performed and evaluate the effectiveness of the Rapid Postoperative Improvement Protocol (RAMPO) in patients undergoing elective gastrointestinal surgery. Method: $A$ total of 122 patients were evaluated, a group of 61 patients received the RAMPO protocol and the other group of equal number of patients were treated with traditional protocols (Controls). Results: Both groups were admitted for colorectal cancer, colostomy due to benign pathology and gastric cancer. The interventions performed were: colorectal resections (35.25\%), intestinal transit restitution (29.51\%) and gastrectomy (15.57\%). In the RAMPO group, postoperative glycemia values (116.49 vs. $167.08 \mathrm{mg} / \mathrm{dl}$ ) reflected better metabolic control with shorter hospital stay (5.49 vs. 14.11 days), obtaining a higher degree of satisfaction (91.80 vs. $19.67 \%)$ when compared to the control group $(p<0.005)$. Conclusion: The RAMPO protocol presented relevant aspects to the traditional management of patients undergoing elective surgeries of the gastrointestinal tract, being safe, acceptable in our environment, with faster hospital discharge, significantly improving the evolution of patients, a lower percentage of complications and a high degree of satisfaction without increasing morbidity and mortality.
\end{abstract}

KEY WORDS: Peroperative. Gastrointestinal surgery. Traditional treatment.

\author{
Correspondencia: \\ ${ }^{*}$ César Ramírez-Cáceres \\ Avda. Ezzio Valerí, Municipio Libertador Fecha de recepción: 19-05-2018 \\ C.P. 5101, Mérida, Venezuela $\quad$ Fecha de aceptación: 24-08-2018 \\ E-mail: cesar.ramirez.cirugia@gmail.com DOI: 10.24875/CIRU.18000470
}

Cir Cir. 2019;87:151-157

Contents available at PubMed www.cirugiaycirujanos.com 


\section{Introducción}

El manejo peroperatorio tradicional de los pacientes intervenidos quirúrgicamente de patología gastrointestinal se ha conducido bajo lineamientos que con frecuencia son adaptados a las condiciones del centro de atención médica, más que en protocolos establecidos en publicaciones científicas, los cuales inciden en el tiempo de restauración de las funciones fisiológicas de cada individuo ante la lesión quirúrgica y la administración de fármacos ${ }^{1}$. El tratamiento peroperatorio es un proceso dinámico que ha ameritado revisiones constantes. Entre los procedimientos tradicionales que anteriormente se consideraban necesarios en la etapa preoperatoria se encuentran la preparación mecánica del intestino, el uso sistemático de sonda nasogástrica y el ayuno prolongado; estos han sido desligados de los procedimientos hasta quedar en desuso ${ }^{2}$. Desde 1971, Nachlas dio a conocer el impacto psicológico que tenía el uso de la sonda nasogástrica en muchos de los pacientes sometidos a cirugía, la cual era empleada con el propósito de prevenir complicaciones como el íleo posoperatorio; no obstante, la exclusión de la sonda en este proceso no representó mayores complicaciones y mostró resultados satisfactorios en la recuperación ${ }^{3}$. Por otra parte, en el posoperatoria, la administración prolongada de sueroterapia, el inicio de la tolerancia oral al reaparecer el peristaltismo, el reposo exhaustivo en cama, y el retiro de catéteres y drenajes, han sido otros factores sometidos a modificaciones, ya que prolongaban la estancia hospitalaria, que oscilaba entre 8 y 12 días $^{2,4}$.

En la necesidad de avanzar en el tema de la recuperación posoperatoria, diversos estudios generaron debates especialmente en cirugía colorrectal, que desde hace cuatro décadas ha sufrido modificaciones satisfactorias en la convalecencia. Dentro de los estudios relevantes se encuentra el de la Universidad de Hvidovre, dirigido por Kehlet ${ }^{5}$ en el Hospital de Dinamarca, en el que se demostraron los beneficios de aplicar estrategias de atención en las distintas etapas del peroperatorio, y se hace alusión a la importancia de informar al paciente del procedimiento que se le realizará, haciéndole consciente de su protagonismo en la recuperación posoperatoria. Posteriormente, Delaney, et al. ${ }^{6}$, en los EE.UU., implementaron los protocolos de dieta y movilización precoz, los cuales permitieron una recuperación más rápida de los pacientes; a estos se les denominó programas de vía rápida o fast-track. Todas estas experiencias se consensuaron en el año 2001 por medio del protocolo ERAS (enhanced recovery after surgery), conformado por diferentes unidades de cirugía colorrectal de países del norte de Europa, como Escocia, Suecia, Dinamarca, Noruega y Holanda ${ }^{7}$. El protocolo ERAS definía la combinación de estrategias preoperatorias, intraoperatorias y posoperatorias cuyo propósito era mejorar la respuesta biológica, minimizar el estrés quirúrgico, disminuir las complicaciones y acortar la estancia hospitalaria, y por ende reducir los costos de hospitalización ${ }^{8}$. En 2005, el protocolo ERAS se amplió en un proyecto conformado por estrategias fundamentales, que se distribuyen en función del momento de actuación ${ }^{7}$, acelerando la recuperación sin incidir en la morbimortalidad de los pacientes ${ }^{5}$. Es imprescindible, para la organización y la implementación óptimas de un régimen peroperatorio, la participación de un equipo multidisciplinario ${ }^{9}$ que esté motivado y que permita alcanzar mejoras significativas en los servicios de los centros de atención médica? ${ }^{7}$.

Basados en la necesidad del entorno hospitalario donde se desarrolló el presente estudio, se consideró pertinente aplicar un protocolo de rápida mejoría posoperatoria (RAMPO) a pacientes intervenidos de patología gastrointestinal de forma electiva ingresados en el servicio de cirugía general del Instituto Autónomo Hospital Universitario de Los Andes, de Venezuela, utilizando como guía las pautas fundamentales del protocolo ERAS, sin incidir en la morbimortalidad.

Los objetivos de este estudio se orientaron a identificar las afecciones gastrointestinales y los tipos de cirugía electiva, y a evaluar la efectividad de la aplicación del protocolo RAMPO.

\section{Método}

En lo relativo a procedimientos de orden metodológico, el estudio se consideró un ensayo clínico controlado en el que se evaluaron 122 pacientes y se distribuyeron en dos grupos: uno conformado por 61 pacientes sometidos al protocolo RAMPO y otro por 61 pacientes a los que se brindó la atención peroperatoria tradicional, con edades comprendidas entre 18 y 86 años, ingresados con patología gastrointestinal para ser intervenidos de forma electiva en el servicio de cirugía general del Instituto Autónomo Hospital Universitario de Los Andes, los cuales se distribuyeron al azar por los especialistas que conforman el equipo del servicio. Se incluyeron pacientes mayores de $18 \mathrm{años}^{10}$, de ambos sexos, con y sin comorbilidad, quedando excluidos aquellos que presentaban patología quirúrgica de emergencia o cardiometabólica descompensada. Se 
Tabla 1. Etapas de preoperatorio, intraoperatorio y posoperatorio del protocolo RAMPO

\begin{tabular}{|c|c|c|}
\hline \multicolumn{3}{|c|}{ PROTOCOLO RAMPO } \\
\hline Preoperatorio & Intraoperatorio & Posoperatorio \\
\hline $\begin{array}{l}\text { Asesoramiento en el servicio de } \\
\text { admisión. } \\
\text { Carga de hidratos de carbono } 2-3 \\
\text { h previas } \\
\text { Ayuno de líquidos } 2-3 \mathrm{~h} \text { previas } \\
\text { Antibioticoprofilaxis } 1 \mathrm{~h} \text { previa } \\
\text { Tromboprofilaxis } 8-12 \mathrm{~h} \text { previas } \\
\text { No preparación ilntestinal }\end{array}$ & $\begin{array}{l}\text { Anestésicos de acción corta } \\
\text { No uso de drenajes } \\
\text { Evitar sobrecarga de líquidos } \\
\text { Normotermia }\end{array}$ & $\begin{array}{l}\text { No sonda nasogástrica } \\
\text { Prevención emesis } 1 \mathrm{~h} \\
\text { Movilización temprana } 4-6 \mathrm{~h} \\
\text { Inicio temprano de tolerancia oral } \\
\text { Deambulacion diaria de } 1 \mathrm{~h} \\
\text { Retiro temprano de catéteres y } \\
\text { drenajes, e inicio de tratamiento } \\
\text { farmacológico por vía oral } \\
\text { Evaluar bienestar del paciente }\end{array}$ \\
\hline
\end{tabular}

registraron datos de variables como la edad, los días de estancia hospitalaria, el diagnóstico clínico, las estrategias preoperatorias e intraoperatorias, la intervención quirúrgica realizada, las estrategias posoperatorias y el control ambulatorio.

Las etapas del manejo peroperatorio tradicional se encuentran descritas en el Manual de diagnóstico y terapéutica quirúrgica de la Universidad de Los Andes (Mérida, Venezuela) ${ }^{11}$, e incluyen:

- Estrategias preoperatorias: ayuno de 12 horas para sólidos y líquidos, antibioticoprofilaxis 2 horas antes de la cirugía, preparación mecánica del intestino (Colyte ${ }^{\circledR}, 2-3$ litros 24 horas antes de la cirugía) y aporte de cloruro de potasio diluido en hidratación parenteral las 24 horas previas a la cirugía.

- Estrategias intraoperatorias: colocación de drenajes y tratamiento anestésico a criterio del servicio de anestesiología.

- Estrategias posoperatorias: administración intravenosa de fármacos analgésicos, opiáceos y antieméticos de ser necesario, deambulación a las 24 horas del posoperatorio, inicio de tolerancia oral al reinstaurar el peristaltismo, retiro de catéteres, hidratación parenteral, drenajes y administración de tratamiento por vía oral el día del egreso hospitalario.

En cuanto al protocolo RAMPO, se establecieron las siguientes estrategias:

- Preoperatoria: asesoramiento e información sobre el peroperatorio, ayuno corto de 2-4 horas para líquidos y 6-8 horas para sólidos, ingestión de una carga de hidratos de carbono 2 horas antes de la cirugía $\left(250 \mathrm{ml} \text { de Gatorade }{ }^{\circledR}\right)^{12-14}$, antibioticoprofilaxis 1 hora previa a la cirugía, no se realiza preparación mecánica del intestino, y tromboprofilaxis 12 horas previas a la cirugía ${ }^{15}$.

- Intraoperatoria: manejo anestésico a criterio del servicio de anestesiología, uso de drenajes si es requerido, evitar sobrecarga de líquidos y mantenimiento de la normotermia ${ }^{15}$.

- Posoperatoria: se descarta el uso de sonda nasogástrica y se retira el catéter vesical precozmente, prevención de náuseas y vómitos, deambulación precoz a las 4-6 horas de la intervención dentro de la habitación ${ }^{14,15}$ y a las 24 horas un mínimo de 1 hora al día, no se usan como analgésicos los opiáceos, inicio de la tolerancia oral con dieta líquida a las 6-8 horas del posoperatorio (independientemente del peristaltismo) ${ }^{13}$, progresión de la dieta a las 24-48 horas del posoperatorio, debiendo instaurarse la dieta habitual a los 4-5 días de la cirugía, acompañado del retiro de catéteres y drenajes, e inicio del tratamiento farmacológico por vía oral ${ }^{15}$. Al cumplir con la deambulación, la tolerancia oral, el control del dolor y la ausencia de fiebre son considerados aptos para el egreso hospitalario. Los puntos del protocolo RAMPO en los que se hizo énfasis quedan establecidos en la tabla 1 , y el orden cronológico de las actividades cumplidas en el protocolo RAMPO se propone en la figura 1.

Es importante destacar que a los dos grupos se les realizó y registró la glucometría 4 horas antes y 2 horas después de la intervención quirúrgica ${ }^{15}$, y se les aplicó un instrumento SERVQUAL ${ }^{16}$ para evaluar el grado de satisfacción con el tratamiento y la atención peroperatoria recibidos. 


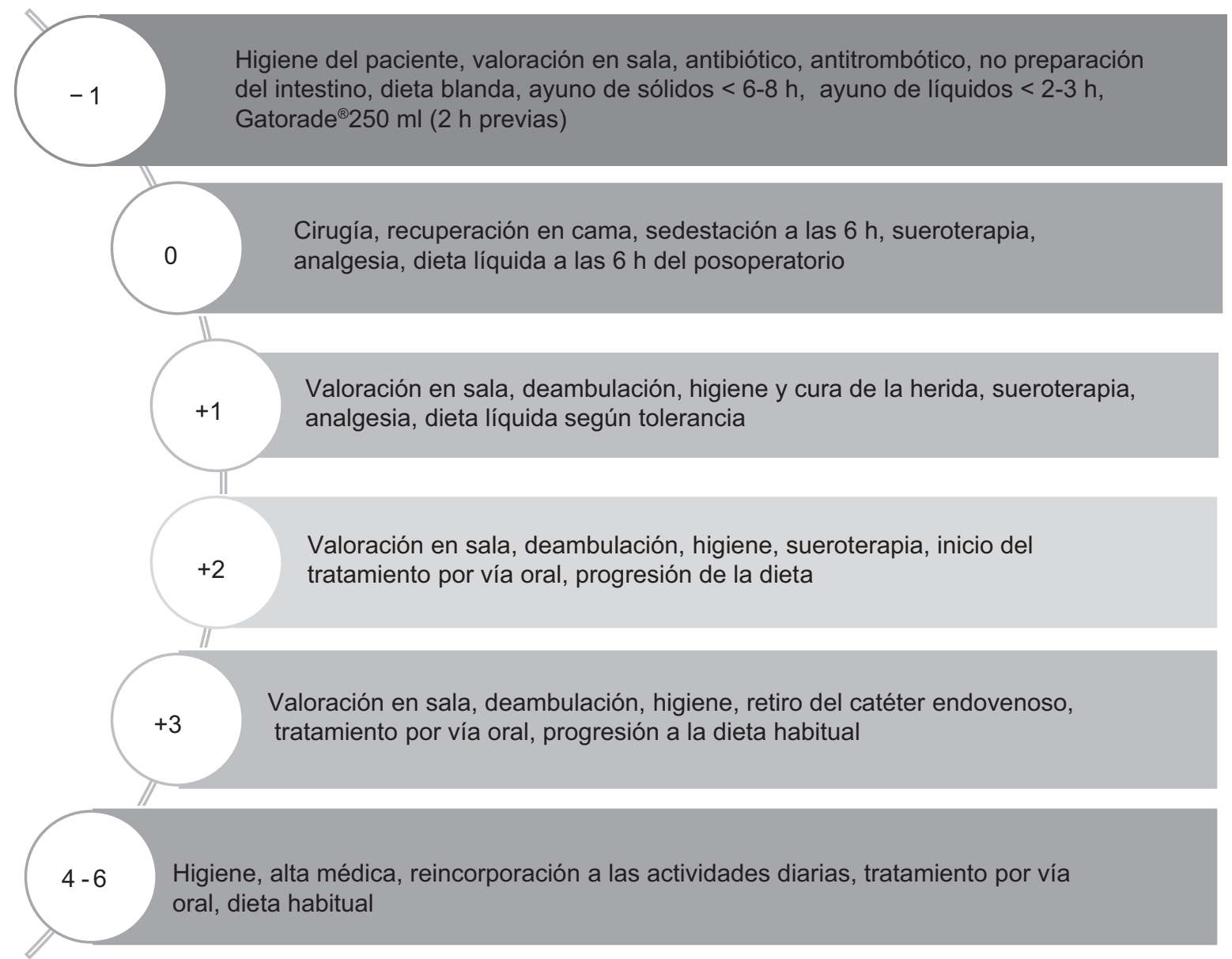

Figura 1. Esquema cronológico del protocolo RAMPO. -1: día previo a la intervención; 0: día de la intervención, recuperación inmediata; $+1,+2$ y +3: días posteriores a la intervención en sala de hospitalización; 4-6: días 4 a 6 de probable egreso hospitalario.

La recolección de datos se realizó mediante la técnica de observación y la entrevista. Para ello, se utilizó una encuesta ad hoc en la que se registraron los datos desde el ingreso hasta los siguientes 30 días posoperatorios, quedando sentados las complicaciones y los reingresos ${ }^{14}$. La base de datos se diseñó en el programa Access. Para la tabulación y el procesamiento de los datos se empleó SPSS versión 15.0. Se aplicó la prueba $t$ de muestras independientes para las variables cuantitativas, y para las cualitativas se realizó la prueba de ji al cuadrado basada en el método estadístico de Fisher; la significación estadística se establecido en 0.05 , y como altamente significativo en 0.005 .

El estudio fue realizado siguiendo los lineamientos establecidos en la declaración de Helsinki ${ }^{17}$ para la investigación en humanos, la confidencialidad de los datos, el consentimiento informado, el Código de Bioética y Bioseguridad del FONACIT'18 y la autorización del comité de ética de la institución.

\section{Resultados}

En ambos grupos los pacientes presentaron una proporción similar en la morbilidad (Fig. 2), observándose con mayor frecuencia patología del tracto digestivo inferior (73.75\%) y superior (26.22\%). Los diagnósticos más frecuentes fueron cáncer colorrectal (35.25\%), ostomías (ileostomía y colostomía) por patología benigna $(22.13 \%)$ y cáncer gástrico (18.03\%). Entre los procedimientos más realizados se encontraron las resecciones colorrectales (33.61\%), la restitución del tránsito intestinal $(29.51 \%)$ y la gastrectomía (15.57\%), seguidas de la cura de prolapso rectal $(8.20 \%)$ y la doble derivación biliodigestiva (3.28\%), los cuales representan el $90.17 \%$ de los procedimientos realizados.

Al comparar la cantidad de líquidos administrados por vía intravenosa en la fase intraoperatoria se observan diferencias significativas: con el protocolo RAMPO se usó un promedio de $1266 \mathrm{ml}$, en contraste con los $1797 \mathrm{ml}$ del método tradicional $(p<0.005)$; no 


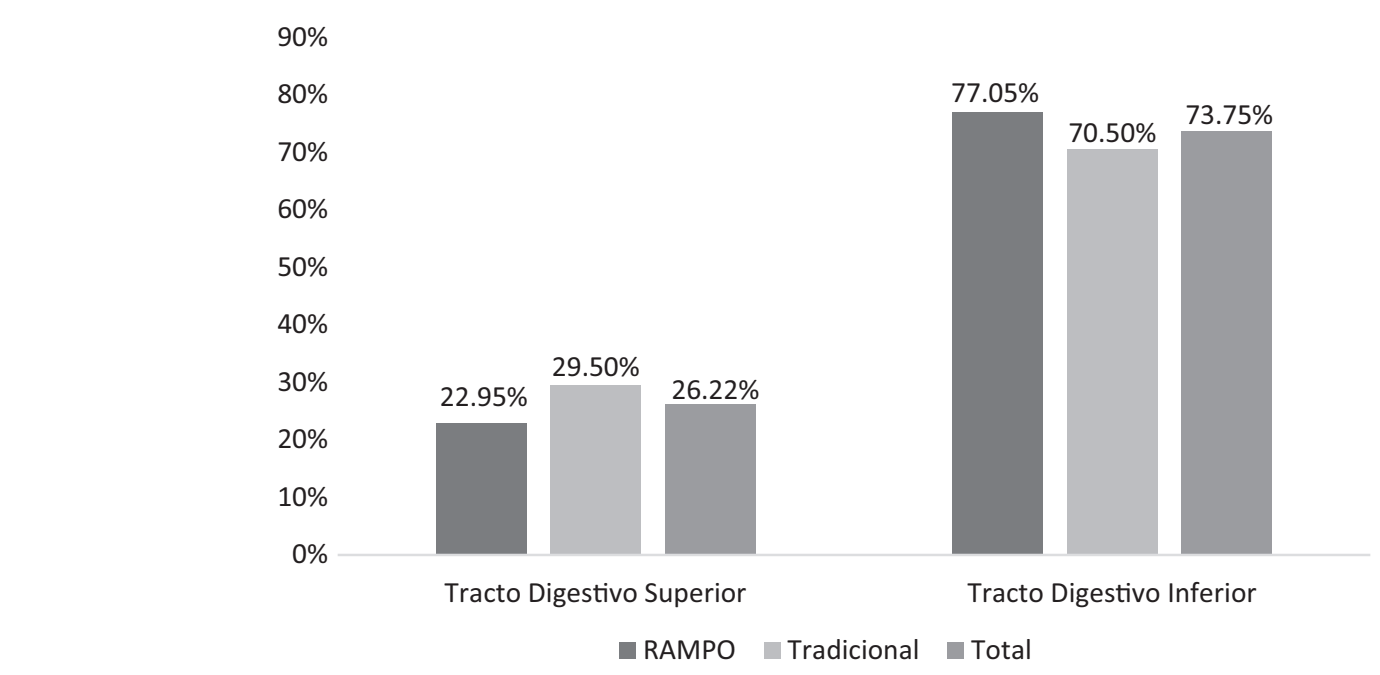

Figura 2. Distribución porcentual de las diferentes afecciones clínicas de los pacientes sometidos a cirugía gastrointestinal distribuidos equitativamente en cada grupo de estudio.

Tabla 2. Tiempo promedio de la etapa posoperatoria observada en los grupos de estudio

\begin{tabular}{lcc}
\hline Variable & RAMPO & Control \\
\hline Deambulación diaria (horas) & 2.6 & 1.5 \\
Inicio de la fase líquida (horas) & 6.2 & 120.5 \\
Progresión a la dieta blanda (días) & 2.4 & 7.3 \\
Inicio de dieta habitual (días) & 3.8 & 10.3 \\
Inicio del tratamiento por vía & 4.6 & 12.9 \\
oral (horas) & & \\
Permanencia de los drenajes (días) & 4.1 & 8.6 \\
\hline
\end{tabular}

hubo diferencias con respecto a la cantidad de sangrado intraoperatorio inherente al procedimiento quirúrgico. En cuanto a los aspectos cualitativos en la fase intraoperatoria, se observó una relación entre el protocolo y la colocación de drenajes $(p<0.005)$ : en el $96.72 \%$ de los pacientes intervenidos mediante el protocolo tradicional se utilizaron drenajes, frente al $78.69 \%$ de los tratados con el protocolo RAMPO. Respecto a la necesidad de transfusión sanguínea, no hubo diferencias entre los grupos.

La cantidad de líquidos intravenosos administrados en el posoperatorio fue, en promedio, de $3287 \mathrm{ml}$ con el protocolo RAMPO y de $13,615 \mathrm{ml}$ con el protocolo tradicional (equivalentes a 1.92 y 6.79 días de tratamiento intravenoso, respectivamente). La deambulación al día promedio fue significativamente mayor con el protocolo RAMPO, así como también fueron más rápidos el inicio precoz de la dieta líquida, la progresión a la dieta blanda, la instauración de la dieta habitual, la suspensión de los medicamentos intravenosos, la administración de tratamiento por vía oral y el retiro de los drenajes, en comparación con los pacientes tratados con el protocolo tradicional (Tabla 2).

Comparando los aspectos cualitativos del grupo RAMPO con los del grupo control en el posoperatorio, se empleó en menor proporción la sonda nasogástrica (1.64 vs. $14.75 \%)$, fue mayor el porcentaje de pacientes con movilización temprana antes de las 8 horas (98.36 vs. $3.28 \%$ ) y presentaron menos complicaciones (8 vs. $46 \%$ ), que fueron distensión abdominal (4 vs. $18 \%$ ), hemorragia digestiva ( 3 vs. $10 \%$ ) y dehiscencia de anastomosis (1 vs. $8 \%$ ) las más observadas con ambos protocolos. No hubo ningún fallecimiento en ninguno de los dos grupos.

El valor promedio de la glucemia previa al preoperatorio en el grupo con protocolo RAMPO no mostró diferencias significativas comparado con el grupo de protocolo tradicional ( $99.66 \mathrm{vs.} 98.97 \mathrm{mg} / \mathrm{dl})$. En cuanto a la glucemia a las 2 horas de posoperatorio, fue significativamente menor (116.49 vs. $167.08 \mathrm{mg} / \mathrm{dl}$; $p<0.005)$. En relación con los días de hospitalización, los pacientes tratados bajo el protocolo RAMPO estuvieron hospitalizados durante un promedio de 5.49 días, frente a 14.11 días los del grupo tratado con el protocolo convencional. Al valorar el grado de satisfacción con el tratamiento empleado para la recuperación en ambos protocolos, se observó una mayor satisfacción por recibir "excelente servicio" en los pacientes bajo el protocolo RAMPO, del $91.8 \%$, frente al $54.10 \%$ de los pacientes con el protocolo tradicional que manifestaron un grado de satisfacción por recibir «buen servicio». 


\section{Discusión}

La instauración de protocolos de manejo multimodal en aras de la rápida recuperación del paciente ha tratado de romper los paradigmas del tratamiento peroperatorio tradicional, partiendo del hecho de la incertidumbre del cirujano por temor a que se presenten complicaciones, viéndose obligado a cambiar una serie de conductas que, si bien en la práctica médica le resultan buenas, prolongan la hospitalización y enlentecen la recuperación del paciente. El protocolo RAMPO tiene un amplio espectro, ya que fue dirigido a pacientes con patología del tracto digestivo tanto inferior como superior, en comparación con estudios $2,7,8,14,15,19$ en los que solo fue desarrollado el protocolo ERAS en patología colorrectal, o como el estudio de Patrón, et al..$^{14}$, en el que se excluyeron los pacientes a los que se realizaron resecciones colónicas sin anastomosis, colectomía con ostomía de protección o cirugía convencional. Coincidimos en que la patología colorrectal es una de las más frecuentes, pero también puede ser aplicado en afecciones del tracto digestivo superior y obtener con ello beneficios, tal como describen Fearon, et al..$^{7}$ y Lui, et al..$^{20}$ en sus estudios.

El protocolo RAMPO destaca la importancia de la participación de un equipo multidisciplinario y hace énfasis en los obstáculos encontrados en el estudio de Pearsall, et al. ${ }^{9}$, como la educación, la mayor comunicación y la colaboración del paciente; todo el personal involucrado está enfocado a identificar las vulnerabilidades del paciente y proporcionarle la información necesaria del procedimiento quirúrgico, sin dejar a un lado la dinámica familiar ${ }^{21}$, disminuyendo de esta manera la angustia psicológica, que es un factor que podría prolongar la recuperación, retardando la cicatrización de las heridas por un notable compromiso de la inmunidad como respuesta al estrés ${ }^{22}$. Otro factor que contribuye al estrés es el ayuno prolongado ${ }^{23}$, por lo que en nuestro estudio se mantuvo una dieta blanda hasta 6 horas antes de la cirugía y los líquidos se cesaron en las 2-3 horas previas a la intervención con la ingesta de $250 \mathrm{ml}$ de Gatorade $^{\circledR}$, que asemeja la carga de hidratos de carbono suministrada por Ljungvist ${ }^{12}$ y Melis, et al. ${ }^{24}, y$ que justifican Velázquez, et al. ${ }^{25}$ en su estudio, en el cual además midieron el volumen residual gástrico, que era menor de $15 \mathrm{ml}$ y evidenciaron que no hubo ningún caso de broncoaspiración.

De los pacientes que fueron tratados con el protocolo RAMPO, ninguno recibió preparación mecánica del intestino ${ }^{19}$, en concordancia con lo publicado por Gustafsson, et al. ${ }^{15}$, quienes destacan los efectos físicos que trae este procedimiento, como la deshidratación que predispone al retardo del peristaltismo después de la cirugía y la predisposición a infecciones y dehiscencia que implican una reintervención. Dentro de las estrategias del protocolo RAMPO no se implementó la administración de ansiolíticos el día previo a la intervención, en contraste con lo descrito por Gustafsson, et al..$^{15}$, quienes justifican la medicación, pero eventualmente podría dar lugar a alteración psicomotora en el posoperatorio, retardando la deambulación y la tolerancia oral.

En las estrategias intraoperatorias, la medicación estuvo bajo el control del servicio de anestesiología, y por razones de disponibilidad no se pudo colocar un catéter epidural a los pacientes, por lo que este estudio difiere de otros publicados $5,7,14,15,19,20,26$.

La elección del abordaje a la cavidad abdominal dependió del tipo de procedimiento. El abordaje laparoscópico fue poco usado por razones de disponibilidad, a pesar de lo descrito por Veenhof, et al. ${ }^{4}$ en su estudio, en el que demostraron que los pacientes muestran una mejor respuesta inmunitaria y una menor respuesta inflamatoria cuando son sometidos a procedimientos mínimamente invasivos, y en otros estudios ${ }^{14,15} \mathrm{se}$ observa que generan menos dolor, por lo que acortan la estancia hospitalaria, sin mayor tasa de reingreso.

El retiro precoz en el posoperatorio del catéter vesical permite que a las 4 horas el paciente pueda iniciar la deambulación, a diferencia de lo publicado por otros estudios ${ }^{15}$ en los que se mantiene durante 1-2 días. Es importante destacar que Patrón, et al..$^{14}$, en su estudio, administraron en el posoperatorio un laxante por vía oral al $67.5 \%$ de los pacientes sometidos al protocolo ERAS, que en nuestro estudio demostramos que no es necesario para una rápida recuperación. De acuerdo con lo descrito por Chalhoub, et al. ${ }^{26}$ y Levis, et al. ${ }^{27}$, se redujo la administración de líquidos y se inició precozmente el tratamiento por vía oral, al igual que el retiro de los drenajes intraabdominales.

En relación con la glucometría posoperatoria, se observaron cambios significativos: las cifras de glucemia en los pacientes tratados con el protocolo tradicional fueron más altas, mientras que los pacientes sometidos al protocolo RAMPO lograron un mejor control metabólico, de modo similar a lo publicado por Velázquez, et al. ${ }^{25}$, quienes achacan al estrés quirúrgico el generar un estado de hipermetabolismo e hipercatabolismo que aumenta la resistencia a la insulina y se manifiesta con hiperglucemia, alterando la respuesta de la cascada inflamatoria e inmunitaria, y de esta manera se incrementa el riesgo de infección nosocomial y se alarga la estancia hospitalaria. El riesgo de complicaciones hospitalarias se ha 
relacionado con la hiperglucemia incluso en pacientes sin antecedentes de diabetes ${ }^{28}$.

En concordancia con algunos estudios, como los de Patrón, et al. ${ }^{14}$ y Launay, et al..$^{10}$, que destacan lo factible y efectivo de la aplicación de protocolos peroperatorios en pacientes de edad avanzada, malnutridos y con comorbilidad, que son un grupo de alto riesgo para prolongar la estancia hospitalaria, paradójicamente son los que más se beneficiarían de un tratamiento multimodal.

En conclusión, el protocolo RAMPO demostró tener efectos relevantes sobre el manejo tradicional de todos los pacientes sometidos a cirugía electiva del tracto digestivo tanto superior como inferior, siendo seguro, aceptable en el medio donde se aplicó, con egreso hospitalario más rápido y mejorando de manera significativa la evolución de los enfermos, con menor porcentaje de complicaciones y un alto grado de satisfacción, sin aumentar la morbimortalidad.

\section{Financiamiento}

No hubo ninguna fuente de financiamiento.

\section{Conflicto de intereses}

Los autores declaran no tener conflicto de intereses.

\section{Responsabilidades éticas}

Protección de personas animales. Los autores declaran que los procedimientos seguidos se conformaron a las normas éticas del comité de experimentación humana responsable y de acuerdo por la asociación médica mundial y la declaración de Helsinki.

Confidencialidad de datos. Los autores declaran que han seguido los protocolos del centro de trabajo sobre la publicación de datos de pacientes.

Derecho de la privacidad y consentimiento informado. Los autores han obtenido el consentimiento informado de los pacientes o sujetos referidos en el artículo. Este documento obra en poder del autor de correspondencia.

\section{Bibliografía}

1. Fuentes DZ, López LS, Salazar DM. Consideraciones de la respuesta fisiológica al estrés quirúrgico. Rev Cubana Anestesiol Reanim. 2014;13: 136-46.
2. Kehlet H. Fast-track colonic surgery: status and prospectives. Recent Results Cancer Res. 2005;165:8-13.

3. Nachlas MM, Younis MT, Roda CP, Wryk JJ. Gastrointestinal motility studies as a guide to postoperative management. Nat Inst Health. 1971;18:510-52

4. Veenhof AA, Vlug MS, Van der Pas MH, Sietses C, Van der Peet DL, De Lange ES, et al. Surgical stress response and postoperative immune function after laparoscopy or open surgery with fast track or standard perioperative care: a randomized trial. Ann Surg. 2012;255:216-21.

5. Kehlet $\mathrm{H}$. Multimodal approach to control postoperative pathophysiology and rehabilitation. Br J Anaesth. 1997;78:606-17.

6. Delaney CP, Fazio VW, Senagore AJ, Robinson B, Halverson AL, Remzi FH. "Fast track" postoperative management protocol for patients with high co-morbidity undergoing complex abdominal and pelvic colorectal surgery. Br J Surg. 2001;88:1533-5.

7. Fearon $\mathrm{KCH}$, Ljungqvist $\mathrm{O}$, Von Meyenfeldt $\mathrm{M}$, Revhaung $\mathrm{A}$, Dejong $\mathrm{CHC}$, Lassen K, et al. Enhanced recovery after surgery: a consensus review of clinical care for patients undergoing colonic resection. Clin Nutr. 2005;24:466-7.

8. Carrillo ER, Espinoza El, Pérez CA. Una nueva propuesta de la medicina perioperatoria. El protocolo ERAS. Rev Mex Anestes. 2013; 36:S296-301.

9. Pearsall E, Meghjt Z, Pitzul K, Asrts M, McKenzie M, Mcleod R, et al. A Qualitative study to understand the barriers and enablers in implementing an enhanced recovery after surgery program. Ann Surg. 2015;261:92-6.

10. Launay SM, Mathonnet M, Theissen A, Ostermann S, Raynaud SA, Slim K. Are enhanced recovery programs in colorectal surgery feasible and useful in the elderly? A systematic review of the literature. J Visc Surg. 2017;154:29-35

11. Uzcátegui $E$, Molina $L$. Manual de diagnóstico y terapéutica quirúrgica. Mérida, Venezuela: Universidad de Los Andes; 2010.

12. Ljungqvist $O$. Insulin resistance and enhanced recovery after surgery. J Parenter Enteral Nutr. 2012;36:389-98.

13. Velázquez GJ. Nutrición enteral precoz en el post operatorio. Rev Latinoam Cir. 2013;1:1-12.

14. Patrón J, Tanoni B, Ruiz H, Cillo M, Bugallo F, Tyrrell C, et al. Protocolo ERAS en cirugía colónica laparoscópica: evaluación de una serie inicial. Rev Argent Cirug. 2015;107:63-71.

15. Gustafsson U, Scott M, Schwenk W, Demartine N, Roulin D, Francis N, et al. Guidelines for perioperatrive care in elective colonic surgery: Enhanced Recovery After Surgery (ERAS) Society recommendations. World J Surg. 2013;37:259-84.

16. Carman JM. Consumer perceptions of service quality: an assessment of the SERVQUAL dimensions. Journal of Retailing. 1990;66:33-55.

17. Declaración de Helsinki de la AMM. Principios éticos para las investigaciones médicas en seres humanos. $64{ }^{\text {a }}$ Asamblea General, Fortaleza, Brasil; 2013.

18. Briceño E, Suárez E, Michelangeli C, Feliciangeli D, Otaiza E, Medible J, et al. Código de bioética y bioseguridad. FONACIT; 2002;2:1-35.

19. Salvans S, Grande L, Gil M. Rehabilitación multimodal en cirugía electiva colorrectal. Barcelona, España: Universidad Autónoma de Barcelona, Hospital del Mar; 2010.

20. Liu XX, Jiang ZW, Wang ZM, Li JS. Multimodal optimization of surgical care shows beneficial outcome in gastrectomy surgery. J Parenter Enteral Nutr. 2010;34:313-21

21. Malley AN, Kenner CA, Kim TI, Blakeney BA. The role of the nurse and the preoperative assessment in patient transitions. AORN J. 2015;102:181-9.

22. Pinto AN, Faiz OM, Davis RA, Almoudaris AL, Vincent $\mathrm{CH}$. Surgical complications and their impact on patients' psychosocial well-being: a systematic review and meta-analysis. BMJ Open. 2016;6:1-23.

23. Zelic M, Stimac D, Mendrila D, Tokmadzic VS, Fiisic E, Uravic, et al. Influence of preoperative oral feeding on stress response after resection for colon cancer. Hepatogastroenterology. 2012;59:1385-9.

24. Melis G, Van Leeuwen P, Blomberg M, Goedhart A, Uitdehaag B, Strack $R$, et al. A carbohydrate-rich beverage prior surgery prevents surgery-induced immunodepression: a randomized, controlled, clinical trial. J Parenter Enteral Nutr. 2006;30:21.

25. Velázquez GJ, Ramírez C, Wix R, Vargas M. Influencia del ayuno preoperatorio precoz sobre la respuesta inmunológica en pacientes sometidos a laparotomía biliar. Rev Vzla Cir. 2008;61:162-70.

26. Chalhoub BY, Álvarez CM, Velázquez GJ. Protocolo ERAS en pacientes sometidos a cirugía electiva. Rev Latinoam Cir. 2013;3:5-11.

27. Levis S, Egger M, Sylvester P. Early enteral feeding versus "nil by mouth" after gastrointestinal surgery: systematic review and meta-analysis of controlled trials. BMJ. 2001;323:153-299.

28. Farrokhi F, Smiley D, Umpierrez GE. Glycemic control in non-diabetic critically ill patients. Best Pract Res Clin Endocrinol Metab. 2011;25:813-24. 